\title{
Approximate Analytical Solutions to the Initial Data Problem of Black Hole Binary Systems
}

\author{
Pedro Marronetti ${ }^{(1)}$, Mijan Huq ${ }^{(2)}$, Pablo Laguna ${ }^{(2)}$, Luis Lehner ${ }^{(1)}$ Richard A. Matzner $^{(1)}$, and \\ Deirdre Shoemaker ${ }^{(2)}$ \\ (1) Center for Relativity, The University of Texas at Austin, TX 78712-1081 \\ (2) Department of Astronomy \& Astrophysics and \\ Center for Gravitational Physics \& Geometry \\ Pennsylvania State University, University Park, PA 16802
}

(October 27, 2018)

We present approximate analytical solutions to the Hamiltonian and momentum constraint equations, corresponding to systems composed of two black holes with arbitrary linear and angular momentum. The analytical nature of these initial data solutions makes them easier to implement in numerical evolutions than the traditional numerical approach of solving the elliptic equations derived from the Einstein constraints. Although in general the problem of setting up initial conditions for black hole binary simulations is complicated by the presence of singularities, we show that the methods presented in this work provide initial data with $l_{1}$ and $l_{\infty}$ norms of violation of the constraint equations falling below those of the truncation error (residual error due to discretization) present in finite difference codes for the range of grid resolutions currently used. Thus, these data sets are suitable for use in evolution codes. Detailed results are presented for the case of a head-on collision of two equal-mass $M$ black holes with specific angular momentum $0.5 \mathrm{M}$ at an initial separation of $10 \mathrm{M}$. A straightforward superposition method yields data adequate for resolutions of $h=M / 4$, and an "attenuated" superposition yields data usable to resolutions at least as fine as $h=M / 8$. In addition, the attenuated approximate data may be more tractable in a full (computational) exact solution to the initial value problem.

PACS number(s): 04.70.Bw,04.25.Dm

\section{INTRODUCTION}

The computation of gravitational wave production from the interaction and merger of compact astrophysical objects is an analytical and computational challenge. These calculations would be able to provide both a predictive and an analytical resource for the gravitationalwave interferometric detectors such as LIGO [1], Virgo and GEO600 20 soon to be online. We concentrate on the case of binary black hole mergers [3].

Besides ours, the other major efforts to numerically simulate black hole coalescences are being carried out by the AEI-WashU-NCSA [4] and the Cornell-Illinois collaborations $[5]$. The resolutions currently under consideration by the AEI-WashU-NCSA effort are as fine as $h=M / 5$ in a computational domain of with $385^{3}$ meshpoints, where $h$ denotes the grid spacing. Our compu- tations are carried out at a similarly modest resolution $h=M / 4$, on $161^{3}$ domains. The goal of all these groups is to perform eventually simulations in domains of up to $1000^{3}$ grid-points, which will allow finer resolution and/or large physical domain sizes.

Regardless of resolution, in order to carry out such simulations, we must construct initial data sets representing binary black hole systems. These data sets should not only satisfy the Einstein constraints but also carry the desired physical content. Binary black hole initial data sets are the subject of this paper.

Under the $3+1$ formulation of General Relativity, the construction of initial data requires solving the Hamiltonian and momentum constraints:

$$
R+\frac{2}{3} K^{2}-A_{i}{ }^{j} A_{j}^{i}=0
$$

and

$$
\nabla_{j} A_{i}{ }^{j}-\frac{2}{3} \nabla_{i} K=0
$$

respectively. Above, $R$ is the 3 -dimensional Ricci scalar constructed from the spatial 3 -metric $g_{i j}$, and

$$
K_{i j}=A_{i j}+\frac{1}{3} g_{i j} K
$$

is the extrinsic curvature tensor. $K$ and $A_{i j}$ are the trace and trace-free parts of $K_{i j}$, respectively. Covariant differentiation with respect to $g_{i j}$ is denoted by $\nabla_{i}$. Spacetime indices will be denoted by Greek letters and spatial indices by Latin letters.

The systematic solution of the constraint equations is due to Lichnerowicz, Choquet-Bruhat, York and others [6]. This procedure involves freely specifying a metric $\tilde{g}_{i j}$, a traceless extrinsic curvature $\tilde{A}_{i j}$ and the trace $\tilde{K}$. It then introduces a conformal factor $\phi$, and a scaling rule to determine the physical $g_{i j}$ and $K_{i j}$ :

$$
g_{i j}=\phi^{4} \tilde{g}_{i j}, \quad A_{i j}=\phi^{-2} \tilde{A}_{i j}, \quad K=\tilde{K}
$$

as functions of the coordinates (defined both in the physical and in the trial conformal space). Solution consists of simultaneously determining $\phi$ (Eq. (i1) becomes an elliptic equation for $\phi$ ) and correcting the longitudinal part of $A_{i j}$. Typically, one introduces a vector potential $w^{i}$ to accomplish this latter task and the problem consists of four coupled elliptic equations for $\phi$ and $w^{i}$ [7]. 


\section{KERR-SCHILD SLICING}

Our work is based on descriptions of black holes in ingoing Eddington-Finkelstein (iEF) coordinates. This choice is motivated by the fact that, in these coordinates, surfaces of constant time "penetrate" the event horizon. Foliations that penetrate the horizon facilitate the excision of the singularity from the computational domain. The essence of black hole excision is the removal of the singularity while preserving the integrity of the spacetime accessible to observers outside the black hole. As originally suggested by Unruh [8], this is only possible if the excised region is fully contained within the event horizon, thus the need to have access to the interior of the black holes.

Other work, such as that of Brügmann [4 for the generic 3-dimensional code, does not use excision, and instead solves $K=0$ initial data in a conformally flat background [9,10]. Here, we keep $K$ non-zero to maintain close similarities to the analytically known single Kerr-Schild black hole. [1]:

The 4-dimensional form of the Kerr-Schild spacetime

$$
d s^{2}=-d t^{2}+d x^{2}+d y^{2}+d z^{2}+2 H\left(x^{\alpha}\right)\left(l_{\lambda} d x^{\lambda}\right)^{2}
$$

describes isolated single black holes. Here the scalar function $H$ has a known form, and $l_{\lambda}$ is an ingoing null vector congruence associated with the solution. For instance for the Schwarzschild solution, $H\left(x^{\alpha}\right)=M / r$, and $l_{\lambda}=\left(1 ; x^{i} / r\right)$, i.e. an inward pointing null vector with unit spatial part. From the fact that Eq. (4) is an exact solution to the Einstein equations, one can write the 3-metric, and the momenta $K_{i j}$ associated with Eq. (4).

Initial data setting for multiple black hole spacetimes using the method described by Matzner, Huq, and Shoemaker (MHS) [7] begins by specifying a conformal spatial metric which is a straightforward superposition of two Kerr-Schild single hole (spatial) metrics:

$$
\begin{aligned}
\tilde{g}_{i j} d x^{i} d x^{j}=\delta_{i j} d x^{i} d x^{j} & +2{ }_{1} H\left(x^{\alpha}\right)\left({ }_{1} l_{j} d x^{j}\right)^{2} \\
& +2{ }_{2} H\left(x^{\alpha}\right)\left({ }_{2} l_{j} d x^{j}\right)^{2} .
\end{aligned}
$$

The fields marked with the pre-index 1 (2) correspond to an isolated black hole with specific angular momentum $\mathbf{a}_{\mathbf{1}}\left(\mathbf{a}_{\mathbf{2}}\right)$ and boosted with velocity $\mathbf{v}_{\mathbf{1}}\left(\mathbf{v}_{\mathbf{2}}\right)$. The superposition of the conformal momenta is defined as follows: The extrinsic curvature for a single hole (say hole 1)

$$
{ }_{1} K_{i j}=\left({ }_{1} \partial_{j} \beta_{i}+{ }_{1} \partial_{i} \beta_{j}-2{ }_{1} \Gamma_{i j 1}^{k} \beta_{k}-{ }_{1} \partial_{t} g_{i j}\right) /\left(2{ }_{1} \alpha\right)
$$

is converted to a mixed-index object,

$$
{ }_{1} K_{i}{ }^{j}={ }_{1} g^{n j}{ }_{1} K_{i n}
$$

The trace of $\tilde{K}$ is calculated as the sum of the corresponding traces:

$$
\tilde{K}={ }_{1} K_{i}{ }^{i}+{ }_{2} K_{i}{ }^{i}
$$

and the transverse-traceless part of the extrinsic curvature $\tilde{A}_{i}^{j}$ as

$$
\tilde{A}_{i}{ }^{j}={ }_{1} K_{i}{ }^{j}+{ }_{2} K_{i}{ }^{j}-\frac{1}{3} \delta_{i}{ }^{j} \tilde{K}
$$

MHS propose the use the metric and extrinsic curvature so defined as a conformal metric and extrinsic curvature to solve the coupled elliptic system [13].

\section{APPROXIMATE SOLUTIONS}

Here we take a different approach. After all, when two black holes are widely separated we expect almost-linear superposition to hold, so the data setting as specified above, without the elliptic solution for $\phi$ and $w^{i}$, should lead to only small errors in the widely separated case. We show here that even for interestingly close separation scenarios, the superposition errors are small, and in fact both the $l_{1}$ and $l_{\infty}$ norms are smaller than those of the truncation error (the discretization error in the calculation) in simulations at currently accessible computational resolutions. In all cases here we present the results for head-on collisions (which are simpler to display) but very similar results are found at similar separations for non head-on data.

Figure 1 shows plots of the norms of the residuals of the Hamiltonian and momentum constraints for a headon collision using initial data $\left\{\tilde{g}_{i j}, \tilde{A}_{i j}\right\}$ provided by Eq. (5) and Eq. (6), respectively. The parameters of the data are $M_{1}=M_{2}=M=1,\left|\mathbf{a}_{1}\right|=\left|\mathbf{a}_{\mathbf{2}}\right|=a=0.5 M$ along the $z$ axis, and the holes are boosted against each other in the $x$ direction with velocity $v=0.5$. The residuals are defined as the absolute value of

$$
\begin{aligned}
\tilde{H} & =\tilde{R}+\frac{2}{3} \tilde{K}^{2}-\tilde{A}_{i}{ }^{j} \tilde{A}_{j}{ }^{i}, \\
\tilde{M}_{i} & =\widetilde{\nabla}_{j} \tilde{A}_{i}{ }^{j}-\frac{2}{3} \widetilde{\nabla}_{i} \tilde{K} ;
\end{aligned}
$$

where again " " " denotes analytic quantities evaluated using the approximate solutions (5) and (6). We see from figure 1 that the violation of the constraints is primarily confined to a region near each hole. This is due to the fact that Eq. (7) is the sum of terms which scale as $O\left(M^{2} / r^{4} \times M / d\right)$, where $r$ is the coordinate distance to the singularity and $d$ is the coordinate separation between holes. For an isolated black hole, these terms cancel each other out exactly, since the Kerr-Schild metric is an exact solution of the constraint equations. However, that is not the case for the spacetime metric and extrinsic curvatures provided by Eqs. (5) and (6). The presence of the perturbation of the second hole in the vicinity of the first hole destroys the balance between these terms, causing the right hand side of Eq. (7) to 
scale as $O\left(M^{2} / r^{4} \times M / d\right)$. As in our computational approach to evolving the spacetime, we mask the interior of the black hole. In this example, we excise the points inside a sphere of radius $a+h$ centered at the hole, where the absolute value of the specific angular momentum $a$ is also the radius of the Kerr ring-like singularity and $h$ is the grid spacing (for figure 1, $h=M / 4$ ). Hence, figure 1 plots precisely the error on the computational domain.

Every finite difference code will have a corresponding truncation error associated with discretization. In most of the finite difference codes under development, this error scales as $O\left(h^{2}\right)$, second order accuracy; therefore, given a second order finite difference discretization of the constraints, the truncation errors are obtained from

$$
\begin{aligned}
H_{t r} & =\bar{R}+\frac{2}{3} \bar{K}^{2}-\bar{A}_{i}{ }^{j} \bar{A}_{j}{ }^{i} \\
M_{i t r} & =\bar{\nabla}_{j} \bar{A}_{i}{ }^{j}-\frac{2}{3} \bar{\nabla}_{i} \bar{K} .
\end{aligned}
$$

Above, "-" is used to denote finite difference discretization, and it is understood that these finite difference expressions are to be evaluated using the exact, not approximate, solutions of the constraints. Of course, these exact solutions are not yet available [14]. In order to circumvent this problem, we obtain an estimate of the truncation errors from the absolute value of

$$
\begin{aligned}
\bar{H} & =\bar{R}+\frac{2}{3} \bar{K}^{2}-\bar{A}_{i}{ }^{j} \bar{A}_{j}{ }^{i}-\tilde{H} \\
\bar{M}_{i} & =\bar{\nabla}_{j} \bar{A}_{i}{ }^{j}-\frac{2}{3} \bar{\nabla}_{i} \bar{K}-\tilde{M}_{i}
\end{aligned}
$$

instead. Although these truncation errors correspond to a modified form of the constraints, these errors should not be substantially different since the structure of the terms involving finite difference operators was not changed, only analytic functions $\left(\tilde{H}\right.$ and $\left.\tilde{M}_{i}\right)$ have been added. Notice that for an isolated hole, the Kerr-Schild spatial metric and extrinsic curvature satisfy exactly the constraint equations $\left(\tilde{H}=\tilde{M}_{i}=0\right)$, leaving us with an error exclusively related to the truncation error. Figure 2 shows the residual $\tilde{H}$ (diamonds) and truncation error $\vec{H}$ (circles) in the Hamiltonian constraint in a region around the rightmost hole of figure 11. The truncation error $\bar{H}$ was obtained in a mesh with grid spacing $h=M / 4$.

In order to compare the global degree of satisfaction of the initial value equations by the methods described here, we calculate the $l_{\infty}$ (maximum) and $l_{1}$ (average of the absolute value) norms of the Hamiltonian and momentum constraints. We do this over the axis joining the black holes from $x=-10 M$ to $x=10 M$, excluding the points inside two segments of length $0.75 \mathrm{M}$, centered around each hole, as indicated above. As shown in Table I, lines 1 and 4 , both the $l_{1}$ and the $l_{\infty}$ norms of the superposition error are less than those of the $M / 4$ truncation error. However figure 2 makes it clear that the truncation error is not uniformly (pointwise) larger than the superposition error. We find in practice that at $M / 4$, superposition yields adequate behavior in the evolution. Figure 3 shows a similar diagram to figure 2 for the momentum constraint. In this case the superposition method can be used to provide data with error pointwise uniformly smaller than the truncation error.

\section{ATTENUATED SUPERPOSITION METHOD}

In order to reduce the residual errors, we propose a variation of the superposition method that preserves the simplicity of being analytical. Essentially, the method consists of multiplying "attenuation" functions into the recipe of the previous section. The new approximate metric $\tilde{g}_{i j}$, trace $K$ and tensor $\tilde{A}_{i}{ }^{j}$ take the form:

$$
\begin{aligned}
\tilde{g}_{i j}^{A}= & \delta_{i j}+2{ }_{1} B\left(x^{k}\right){ }_{1} H\left(x^{k}\right){ }_{1} l_{i}{ }_{1} l_{j} \\
& +2{ }_{2} B\left(x^{k}\right){ }_{2} H\left(x^{k}\right){ }_{2} l_{i}{ }_{2} l_{j}, \\
\tilde{K}^{A}= & { }_{1} B{ }_{1} K_{i}{ }^{i}+{ }_{2} B{ }_{2} K_{i}{ }^{i}, \\
\tilde{A}_{i}^{A}{ }^{j}= & { }_{1} B{ }_{1} K_{i}{ }^{j}+{ }_{2} B{ }_{2} K_{i}{ }^{j}-\frac{1}{3} \delta_{i}{ }^{j} \tilde{K}^{A} .
\end{aligned}
$$

The purpose of the attenuation function $B$ is to minimize the effects due to a given hole on the neighborhood of the other hole. For instance, the attenuation function ${ }_{1} B$ is unity everywhere except in the vicinity of hole2 where it rapidly vanishes so the metric and extrinsic curvature there are effectively that of a single black hole. The attenuation functions with this property can be constructed in a number of different ways. An example of an attenuation function is

$$
{ }_{1} B=1-e^{-r^{4} / \sigma^{4}}
$$

where

$$
\begin{aligned}
r^{2} & =\frac{1}{2}\left(\rho^{2}-a^{2}\right)+\sqrt{\frac{1}{4}\left(\rho^{2}-a^{2}\right)^{2}+a^{2} z^{2}}, \\
\rho & =\sqrt{{ }_{2} \gamma^{2}\left(x-{ }_{2} x\right)^{2}+\left(y-{ }_{2} y\right)^{2}+\left(z-{ }_{2} z\right)^{2}} .
\end{aligned}
$$

Here ${ }_{2} x^{i}, a$ and ${ }_{2} \gamma$ denote the coordinate location, specific angular momentum and boost factor of hole-2, respectively. $\sigma$ represents a free parameter of the attenuation function. An expression similar to (13) for ${ }_{2} B$ is obtained by reversing the labels.

The attenuation function (13) was chosen amongst a few different types for its simplicity and better performance. Since the constraints involve second order derivatives, it is important to pick attenuation functions that vanish up to second order derivatives, as in (13), so "pure" single black hole solutions can be obtained in the neighborhood of each hole. 


\section{RESULTS}

Figures 2 and 13 and 5 ) show the Hamiltonian (momentum) constraint for a region around the rightmost hole of figure 1 . Figure 2 shows a comparison between the truncation error for $h=M / 4$ (empty circles) and the superposition initial data (full diamonds), while figure 1 shows the same for attenuated initial data (full squares).

First we note in figure that in some areas (near $x=2 M$, for instance) the violation of the constraints for these attenuated data is greater than that corresponding to truncation error. However, even in these areas the violation is smooth and small in absolute value, as opposed to the unbounded behavior shown by the superposition data near the singularity, and the divergent behavior present at the singularity has disappeared with the use of attenuated data. This is due to the fact that, having cancelled the influence of the presence of the second hole, the fields $\tilde{g}_{i j}^{A}$ and $\tilde{K}_{i j}^{A}$ become the fields corresponding to an isolated black hole at the location of the singularity.

The results are presented in Table I. The momentum constraint $l_{1}$ norm represents the average of the three components of Eq. (8) and the $l_{\infty}$ the maximum value of them. These values are compared to the norms of the truncation error in the constraints caused by the finite difference stencils for grid spacings $h=M / 4, M / 8$, and $M / 16$. These results show that with these norms, the superposition initial data violate the Hamiltonian and momentum constraints below the truncation error corresponding to grid spacings as fine as $h=M / 4$, while the attenuated data extend this range to at least $h=M / 8$. Note also that, for both the superposition and the attenuated method, the momentum constraint equations are satisfied well within the truncation error for grid spacings as fine as $h=M / 16$. Finally Table 1 (rows 2, 3) shows the approximate second order convergence between resolutions $h=M / 8$ and $h=M / 16$. The convergence rate is approximately second order because even at $h=M / 16$, finite $h$ effects affect significantly the convergence.

\section{CONCLUSIONS}

The initial value problem for black hole binary systems is currently approached by solving numerically a set of elliptic equations derived from the Hamiltonian and momentum constraint. Due to the presence of singularities in the fields, these equations are complex and difficult to handle.

In this article we presented analytical methods that can provide initial value data for black hole binary systems. While these solutions are only approximate, they satisfy the Hamiltonian and momentum constraint equations to the level of accuracy (evaluated with the $l_{1}$ and $l_{\infty}$ norms) of the truncation error present in finite difference codes for a given range of grid resolutions. Because attenuated data are so much smoother than the superposition, and because the principal difficulties in computational simulations arise from sharp gradients and divergent values, which the attenuation method eliminates, we are confident that at least for the particular example of a head-on collision of two equal-mass black holes at a separation of $10 M$, thess data could be used in evolutionary codes with grid spacings as fine as $h=M / 8$.

The analytical nature of these methods makes them simpler to implement than the numerical approach. Furthermore, a more comprehensive study of the attenuation functions may extended the range of resolutions for which this approximation is valid.

The attenuation method offers the advantage that it cancels the divergent behavior of the constraints near the central singularity. The suppression of contributions near the black holes (individual holes have exactly zero residuals) should simplify the exact problem, since this method provides exact inner boundary conditions for the elliptic solver.

\section{ACKNOWLEDGEMENT}

This work was supported by NSF grant PHY9800722 and PHY9800725 to the University of Texas at Austin and PHY9800973 and PHY9800970 to the Pennsylvania State University.

[1] A. Abramovici, et al., Science 256, 325 (1992).

[2] C. Bradaschia, et al., Nucl. Instrum. Meth., Phys. Res. Sect., 289, 518 (1990).

[3] The Binary Black Hole Grand Challenge Alliance: Gomez,R., et al., Phys. Rev. Lett. 80, 3915 (1998).

[4] B. Brügmann, Int. J. Mod. Phys. D8, 85 (1999).

[5] Scheel, M., et al., Physical Review D56, 6320 (1997).

[6] J. W. York and T. Piran "The Initial Value Problem and Beyond", Spacetime and Geometry: The Alfred Schild Lectures, R. A. Matzner and L. C. Shepley Eds. University of Texas Press, Austin, Texas. (1982); G. B. Cook, "Initial Data for the Two-Body Problem of General Relativity", Ph.D. Dissertation, The University of North Carolina at Chapel Hill (1990).

[7] R. A. Matzner, M. F. Huq, and D. Shoemaker, Physical Review D59, 024015 (1999).

[8] J. Thornburg, Class. Quantum Grav., 4, 1119-1131 (1987).

[9] S. Brandt and B. Brügmann, Phys. Rev. Lett. 78, 3606 (1997).

[10] G. B. Cook, M. W. Choptuik, M. Dubal, S. A. Klasky, 
R. A. Matzner and S. R. Oliveira, Physical Review D, 1471-1490 (1993).

[11] R. P. Kerr and A. Schild, "Some Algebraically Degenerate Solutions of Einstein's Gravitational Field Equations" Applications of Nonlinear Partial Differential Equations in Mathematical Physics, Proc. of Symposia B Applied Math., Vol. XV11, (1965).

"A New Class of Vacuum Solutions of the Einstein Field Equations," Atti del Convegno Sulla Relativita Generale: Problemi Dell'Energia E Onde Gravitazionale, G. Barbera, ed.,(1965).

[12] For this article we work with the constraint functions instead of their normalized counterparts. The normalized constraints provide the degree of violation compared to the magnitude of the terms in each equation and can be misleading when these terms have a relatively small absolute value.

[13] The final step in the definition of the initial transversetraceless extrinsic curvature tensor consists in the construction of the symmetric tensor $\tilde{A}_{i j}=\tilde{g}_{n(i} \tilde{A}_{j)}^{n}$.

[14] Marronetti et al., Work in preparation (2000).

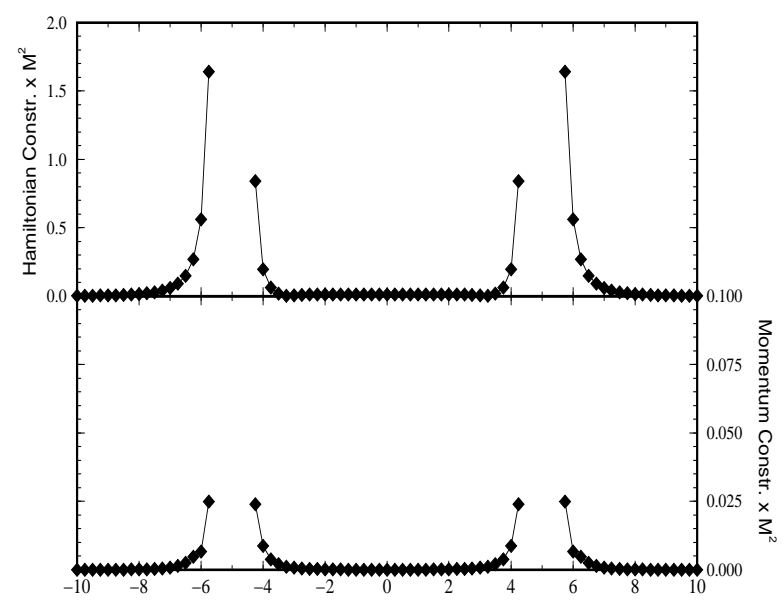

FIG. 1. Hamiltonian and momentum constraint residuals for a head-on collision of two equal-mass $M$ black holes. The vertical axis shows the residuals of the Hamiltonian constraint $|\tilde{H}|$ and the $x$ component of the momentum constraint $\left|\tilde{M}_{x}\right|$ defined by Eqs. (0,8). The black holes are separated $10 M$ and boosted toward each other at $0.5 c$. Both black holes have specific angular momentum $0.5 M$ pointing perpendicular to the colliding direction. The momentum residual corresponds to the component parallel to the colliding axis.

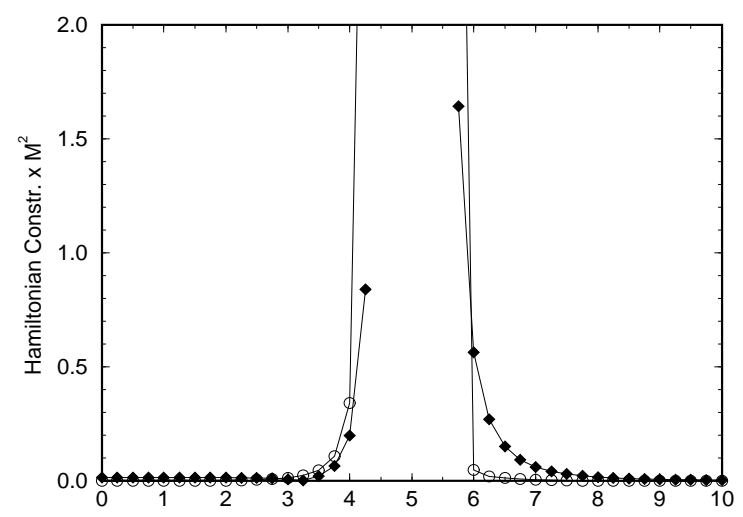

FIG. 2. Hamiltonian constraint near the rightmost hole for the system of figure 1. The diamonds show the Hamiltonian constraint residuals $|\tilde{H}|$ for the superposition method. The circles are the estimation $|\bar{H}|$ of the truncation error present in a second order finite difference code $(h=M / 4)$, defined by Eq. (11).

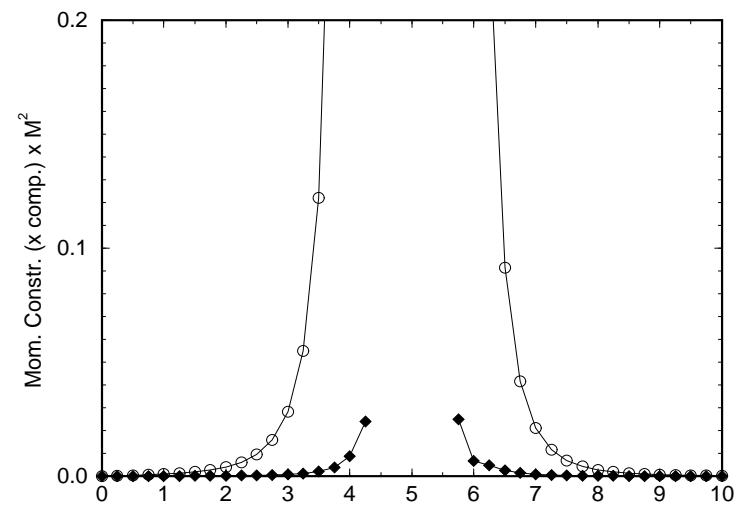

FIG. 3. Momentum constraint (x component) for the system of figure 2. The diamonds show the momentum constraint residuals $\left|\tilde{M}_{x}\right|$ for the superposition method. The circles are the estimation $\left|\bar{M}_{x}\right|$ of the truncation error $(h=M / 4)$, defined by Eq. (12). 


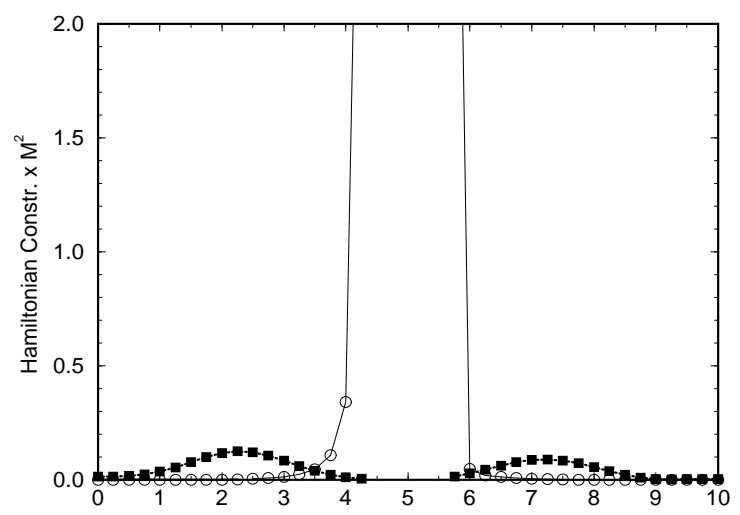

FIG. 4. Hamiltonian constraint for the system of figure 1 . The squares present the Hamiltonian constraint residuals $|H|$ for the attenuation method. The circles are the estimation $|\bar{H}|$ of the truncation error present in a second order finite difference code $(h=M / 4)$, defined by Eq. (11).

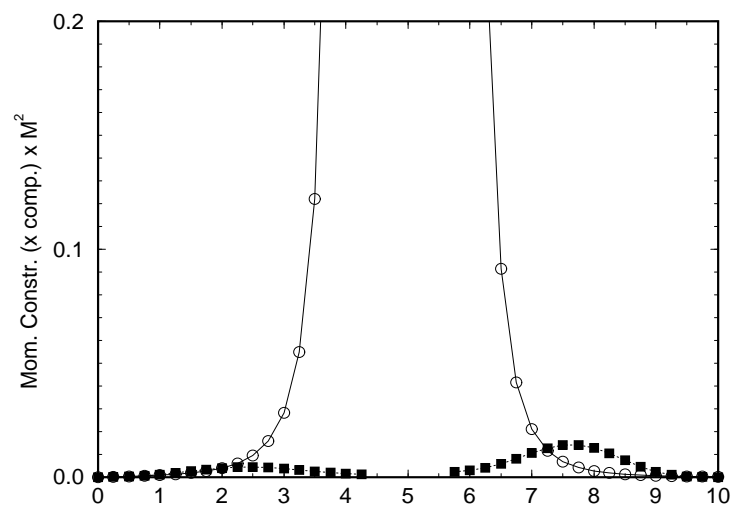

FIG. 5. Momentum constraint (x component) for the system of figure 4 . The squares show the momentum constraint residuals $\left|\tilde{M}_{x}\right|$ for the attenuation method. The circles are the estimation $\left|\bar{M}_{x}\right|$ of the truncation error $(h=M / 4)$, defined by Eq. (12).

TABLE I. Truncation errors (first three rows) and residuals (last two rows) of the Hamiltonian and momentum constraints.

\begin{tabular}{lcccc}
\hline \hline Method & \multicolumn{2}{c}{ Ham. Constr. } & \multicolumn{2}{c}{ Mom. Constr. } \\
& $l_{\infty}$ & $l_{1}$ & $l_{\infty}$ & $l_{1}$ \\
\hline & & & & \\
$h=M / 4$ & 4.240 & 0.251 & 20.20 & 0.605 \\
$h=M / 8$ & 0.757 & 0.022 & 3.098 & 0.065 \\
$h=M / 16$ & 0.198 & 0.004 & 0.742 & 0.012 \\
without attenuation & 1.643 & 0.097 & 0.235 & 0.003 \\
with attenuation & 0.126 & 0.049 & 0.041 & 0.005 \\
\hline \hline
\end{tabular}

